\title{
HIERARCHICAL SPATIAL REASONING APPLIED TO SPATIAL DATA INFRASTRUCTURES
}

\author{
Abbas Rajabifard*1, Francisco Escobar ${ }^{2}$ and Ian P. Williamson ${ }^{3}$ \\ Department of Geomatics, The University of Melbourne, Victoria 3010, Australia \\ Phone: +61 383444431 Fax: +61 393474128 \\ http://www.geom.unimelb.edu.au/research/SDI_research/ \\ ${ }^{1}$ abbas@sunrise.sli.unimelb.edu.au \\ ${ }^{2}$ f.escobar@eng.unimelb.edu.au, ${ }^{3}$ i.williamson@eng.unimelb.edu.au
}

\begin{abstract}
Many countries throughout the world believe they can benefit both economically and environmentally from better management of their spatial data assets, enabling them to access and retrieve complete and consistent datasets in an easy and secure way. This has resulted in the development of the Spatial Data Infrastructure (SDI) concept at various political and/or administrative levels. The SDI concept has been represented by different descriptions of its nature, however, currently these demonstrate an overly-simplistic understanding of the concept.

The simplicity in existing definitions has been slow to incorporate the concept of an integrated, multilevelled SDI formed from a hierarchy of inter-connected SDIs at corporate, local, state/provincial, national, regional (multi-national) and global (GSDI) levels. Failure to incorporate this multidimensionality, and the dynamic mechanistic and functional roles of the SDI, have rendered many descriptions of SDI inadequate to describe the complexity and the dynamics of SDI as it develops, and thus ultimately constrain SDI achieving developmental potential in the future.
\end{abstract}

As a result, the objective of this paper is to demonstrate the fitness and applicability of Hierarchical Spatial Reasoning (HSR) as a theoretical framework to demonstrate the multi-dimensional nature of SDIs. It is argued that by better understanding and demonstrating the nature of an SDI hierarchy, any SDI development can gain support from a wider community of both government and nongovernment data users and providers. The findings presented in this paper build on the authors' experiences in Regional SDI (multi-national) development and HSR.

\section{INTRODUCTION}

Current progress for SDI initiatives throughout the world show that after many years of effort these initiatives still do not receive support from all community members, depending on the political/administrative level to which they belong. Despite the interest and activities in SDI development, it remains very much an innovative concept among members of different communities. For example, after years of effort from the Permanent Committee on GIS Infrastructure for Asia and the Pacific (PCGIAP) on the Asia-Pacific Spatial Data Infrastructure (APSDI), and the European Umbrella Organisation for Geographic Information (EUROGI) on the European Geographic Information Infrastructure (EGII), the first two Regional SDI initiatives, these still receive incomplete support from all respective member nations and organisations (Mohamed 1999, Longhorn 2000).

This problem of limited support is observed in many NSDI initiatives throughout the world as well (Masser 1998, Onsrud 1998). Based on the recommended organisational model for the long-term development of the GSDI, and current difficulties faced by many SDI initiatives, it was highlighted 
by Rajabifard et al. (2000) that the GSDI initiative is going to face the same challenges as other SDI initiatives in its future development and implementation phases. Reasons suggested for the limited support from certain nations, regional organisations and other relevant institutions, include lack of awareness of the value of SDIs, the incompatibility of the current conceptual and organisational model with the perceived needs of the respective communities, and the complexity of different issues such as diverse political, cultural and economical positions.

One way to optimise support is to increase the level of understanding and awareness of people (both users and producers of spatial data, and concerns of relevant politicians) about the nature and the value of SDI concepts in general, and the relationships between different levels of SDIs in particular. HSR provides an expandable framework to demonstrate the concept of SDI and represent complexities of the different SDI levels based on hierarchical principles. This provides a model of the flow on effects achievable through SDI by embracing a hierarchical concept.

This paper attempts to demonstrate the hierarchical relationships between different political/administrative levels of SDIs. It explores the applicability of HSR as a theoretical framework to describe the multi-dimensional nature of SDIs in which to explore the benefits of this hierarchy to increase awareness of SDIs. However, due to the complex nature of SDIs, this paper also attempts to show that current properties of HSR are insufficient to demonstrate fully the dynamic and multi-dimensional nature of SDIs. With this aim, this paper will review the concept of spatial hierarchy and its properties and then discuss the concept of an SDI hierarchy by introducing different views on the nature of this hierarchy. The paper concludes by examining how current hierarchical theory can be extended to incorporate different levels of SDI initiatives.

\section{HIERARCHICAL SPATIAL THEORY}

In the past much research has been conducted toward maximising the efficiency of computational processes by using hierarchies to break complex tasks into smaller, less complex tasks (Car 1997, Timpf 1998). Hierarchical principles are used in many different disciplines to break complex problems to sub problems that can be solved in an effective manner. Examples of hierarchical applications include classification of road networks (Car 1997), development of political subdivisions and land-use classification (Volta and Egenhofer 1993). The complexity of the spatial field as highlighted by Timpf (1998) is primarily due to space being continuous and viewed from an infinite number of perspectives at a range of scales.

\section{Definition of Hierarchy}

Koestler (1968), as cited by Car (1997), used the term hierarchy for a tree-like structure of a system which can be subdivided into smaller sub-systems, which in turn can be further subdivided into smaller sub-systems, and so on. In Figure 1, an example of a hierarchical structure is given, where each new square can be divided into a set of four smaller squares. ABCD consists of four subsquares. This can be recursively subdivided as long as subdivision makes sense. This hierarchical arrangement can also be represented as a tree. 

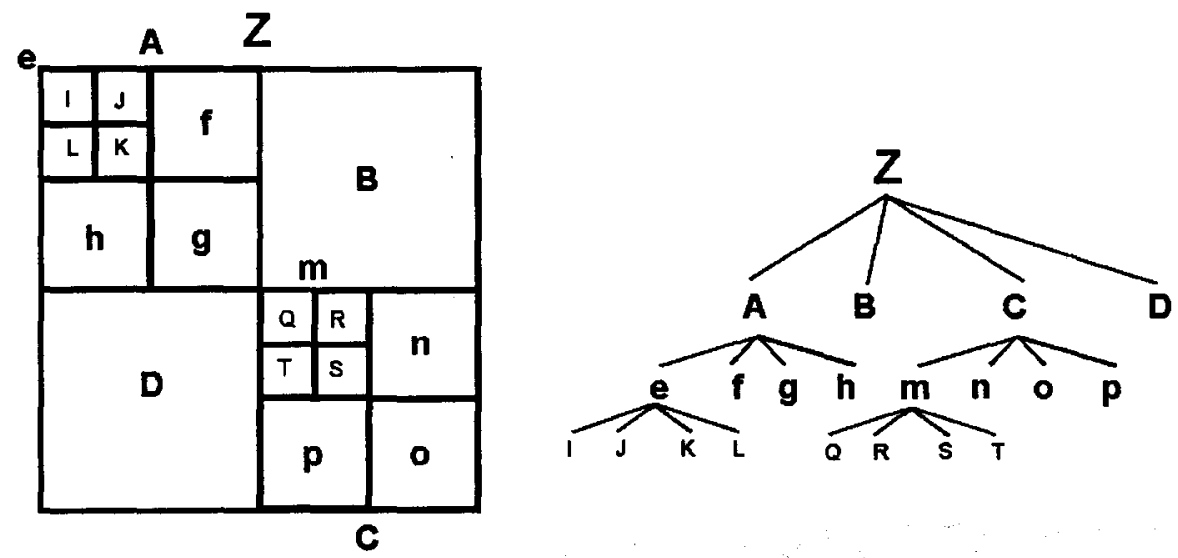

Figure 1. Hierarchical structures represented by square subdivisions and by a tree like structure (adapted from Car, 1997)

Hierarchies are usually distinguished by their functions, which produce different types of hierarchies. Timpf (1998) recognised aggregation, generalisation and filtering as the three most important functions to produce three different types of hierarchies. The aggregation hierarchy is built by aggregating objects. The generalisation hierarchy defines how classes are related to more generic super, or higher order, classes. The filter hierarchy filters objects according to a criterion.

\section{Purpose and Levels of a Hierarchical Structure}

There are good reasons why hierarchies develop and persist. Decreasing the processing time (Pattee 1973, Car 1997) is one reason to introduce a hierarchy into a system: a process being a sequence of actions performed in a particular way and leading to some result, and the processing time is thought of as time needed either for development or evolution of a system. A hierarchically structured system evolves much faster than a non-hierarchical system containing the same number of elements (Simon 1973). Increasing the stability of any system is another reason to form hierarchies (Pattee 1973). Also, hierarchies break down the task into manageable portions, and enhance the potential for parallel processing (Timpf et al. 1992). The hierarchical approach was especially adopted in the description of complex dynamic systems (Mesarovic et al. 1970 as cited by Timpf 1998), which Simon (1981) states have several advantages to a hierarchical structure.

With regard to the levels in a hierarchical structure, a set is divided into subsets or levels. A level is described by criteria determining which elements of the initial set belong to this level, and in turn, how this level is related to other levels in a hierarchy. The number of levels determines the depth of the hierarchy. The number of elements on each level determines its span and in turn the span of the tree.

\section{Hierarchical Reasoning}

Hierarchical reasoning is any reasoning process that applies hierarchy either to sub-divide the task, problem, process or space. Hierarchical reasoning adopts the principle of using the least detailed representation to answer a question. All data are inherently imprecise, but decisions do not require perfect information, instead information that is sufficiently precise (Timpf and Frank 1997).

Hierarchical Spatial Reasoning (HSR) is defined by Car (1997) as part of the spatial information theory that utilises the hierarchical structuring of space for efficient reasoning. It is only recently, through the works of Car (1997) for way-finding, Glasgow (1995) for spatial planning and Frank and Timpf (1994) devising the intelligent zoom, that this theory has started to be applied in the spatial industry. 


\section{Principles of HSR}

The framework supporting HSR has three important components - representation, properties and applications. Hierarchies have been represented using alternative methods: Coffey (1981) devised triangles to represent a hierarchical structure; Car (1997) illustrates how triangles can also be represented as a tree-like structure. Although there are different representations of hierarchically organised systems, all provide the same function to breakdown the complexity of problems into smaller sub systems that can be efficiently handled and modelled.

In the past HSR research has focused on zero and one-dimensional structures to model urban systems (as points), road and drainage networks (as lines), and to a certain extent, to model simple bidimensional objects such as square polygons in quad-trees. Recently, research on HSR has focused on three-dimensional structures to break down the complexities of polygons in the case of Australian administrative boundary design (Eagleson et al. 1999). From this research it has become evident that the properties required to model polygon hierarchy are more complex than those utilised for the modelling of points or networks.

\section{Properties of Hierarchies}

Hierarchies in various phenomena, both natural and artificial, have properties specific to a particular context, but they also have common properties. These common properties are general relationships among structure, movement and function that are independent of their specific context (Car 1997). Some of the properties of a hierarchical structure that are relevant to the understanding of hierarchies in general, and spatial hierarchies in particular, are as follows:

- Part-Whole Property. In a hierarchy, an element on a higher level consists of one or more elements on the lower level. In view of a part-whole relationship, a higher level is a whole and a lower element is its part (Car 1997). For example, in Figure 1, quadrangle A is a whole made up of quadrangles $e, f, g$, and $h$. Similarly, $A$ is also part of quadrangle $Z$.

- Janus-Effect. An element at a hierarchical level has two different faces, one looking toward wholes in a higher level and the other looking toward parts in a lower level. This property was introduced by Koestler (1968, cited by Car 1997) as a fundamental property of all types of hierarchy. In Figure 1, each quadrangle is directly related to both above and below level quadrangles. Thus, e faces $\mathrm{A}$ but also I, J, K, and L.

- Near Decomposability. The third fundamental property of hierarchy is called near decomposability (Simon 1973). It is related to the nesting of systems within larger sub-systems, and is based on the fact that interactions between various kinds of systems decrease in strength with distance. Components that are closer to each other interact more strongly than components that are far apart, many of them being at the same level. The definition of this property does not refer to whether elements on the same level should or should not be closer and have more interaction than elements in other levels. In Figure 1, elements such as $\mathrm{J}$ or $\mathrm{K}$ are closer to $\mathrm{A}$ than to other elements on the same level such as $\mathrm{T}$ or $\mathrm{Q}$. In the tree structure part of the same diagram, it is clear how elements within the same level do not necessarily interact with themselves. It is believed, and will be discussed later, that elements within the same level in the hierarchy should have a way to communicate or interact in a better way than what is already present amongst levels.

Other than properties, hierarchies may also have special functional features such as uniqueness in particular roles. A feature such as this uniqueness may distinguish one level of hierarchy due to its inter-relatedness with the other levels of hierarchy. This feature is known as particularity to the system of hierarchies. 


\section{SPATIAL DATA INFRASTRUCTURE}

Spatial Data Infrastructure (SDI) is an initiative intended to create an environment that enables a wide variety of users, who require access to and retrieval of consistent data sets, of a certain area covered by the SDI, in an easy and secure way. It can also be viewed as a tool to provide an environment in which all stakeholders, both users and producers of spatial data, can cooperate with each other and utilise technology in a cost-effective way to better achieve the objectives at the appropriate political/administrative level.

SDIs are now recognised as a central component in supporting an information society. Countries and states are committed to finding workable strategies and models to support SDI initiatives due to the potential that SDIs offer for managing our natural and built environment in an information society.

SDIs have become a crucial tool in determining the way in which spatial data are used throughout an organisation, a nation, different regions and the world. They allow the sharing of data, which enables users to save resources, time and effort when trying to acquire new data sets by avoiding duplication of expenses associated with generation and maintenance of data and their integration with other data sets. Central to achieving efficient and effective use of spatial information to meet organisational and business objectives is thus optimising the potential of SDIs to support spatial information interactions. However, to empower SDI framework implementation and SDI optimisation in the spatial information industry, technical and institutional issues of access to useable data need to be addressed.

SDI is defined differently by many stakeholders trying to capture the nature of SDI. For example, the Federal Geographic Data Committee (FGDC 1997) defines the United States' national SDI as an umbrella of policies, standards, and procedures under which organisations and technologies interact to foster more efficient use, management, and production of geospatial data. It further explains that SDIs consist of organisations and individuals who generate or use geospatial data and the technologies that facilitate use and transfer of geospatial data. Another example is the Australian and New Zealand Land Information Council (ANZLIC 1998) that defines a national SDI as comprising four core components: an institutional framework, technical standards, fundamental datasets, and clearing house networks.

These definitions together with other attempt (McLaughlin and Nichols 1992, Executive Order 1994, European Commission 1995, GSDI 1999) provide a useful base for understanding of SDI. But, individually they are unable to demonstrate different aspects of SDIs. Individual SDIs are designed to meet the criteria defined by stakeholders from particular communities. While these criteria meet the objectives of the immediate SDI, they remain isolated unless integrated with the objectives of related SDIs and the broader SDI network. In other words, current understandings of SDI have resulted in fragmentation of the objectives and nature of SDI, which has limited the ability to adapt SDI evolution. This is also reflected in baseline information provided by Onsrud (1998) on the nature and characteristics of SDIs development throughout the world.

Due to this criticism, SDI remains very much an innovation even among SDI practitioners and there remain doubts regarding the nature and identities of SDI (Barr 1998, Rajabifard et al. 2000). For example, after many years of coordination and implementation of the US-National SDI, by the FGDC, and efforts from other committees, such as the Mapping Science Committee (MSC), the development of the National SDI is still challenged by lack of support from some member states and is faced with many implementation difficulties. In the Asia-Pacific region, after more than six years of efforts on the development of the APSDI, still this initiative does not receive support from all member nations and regional organisations (Mohamed 1999). This is emphasised by the generally limited understanding of the innovative concepts of SDI even among key players in the spatial information industry (Barr 1998, 1999, Coleman and MacLaughlin 1998), and the complexity of the relationships between different SDIs initiatives in a particular political/administrative level. Greater 
understanding of the concept of SDI can be obtained from a more functional model of the nature, capacity and relationships, sustaining SDI and SDI development. An SDI hierarchy provides a model with such functionality.

\section{SDI HIERARCHY}

As a result of developing SDIs at different political/administrative levels, a model of SDI hierarchy that includes SDIs developed at different political-administrative levels was developed and introduced (Chan and Williamson 1999, Rajabifard et al. 2000). Figure 2 illustrates this model in which an SDI hierarchy is made up of inter-connected SDIs at corporate, local, state/provincial, national, regional (multi-national) and global levels. In the model, a corporate GIS is deemed to be an SDI at the corporate level-the base level of the hierarchy (Chan and Williamson 1999). Each SDI at the local level or above is primarily formed by the integration of spatial data sets originally developed for use in corporations operating at that level and below.

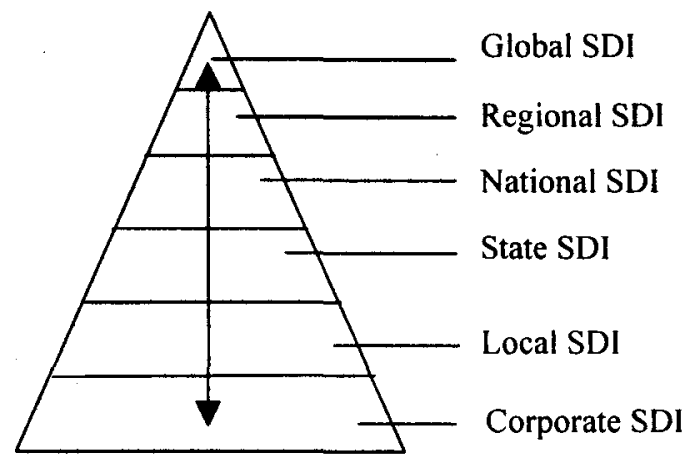

Figure 2. An SDI Hierarchy of SDIs at different levels of Jurisdictions

The main reason that a hierarchy concept is applied is that all common properties and reasons for developing a hierarchical structure are also applicable to SDI concepts. For example, according to the part-whole property, an SDI on a higher level, like a global level, consists of one or more SDIs on the lower level, such as different Regional SDIs like the APSDI in the Asia-Pacific and the EGII in Europe. Moreover, a Regional SDI is a whole for a regional level and is a part of the global level. This is also applicable to the individual components of an SDI. Alternatively, according to the JanusEffect, any element at a hierarchical level, say a National SDI, in the SDI hierarchy has two different faces, one looking toward wholes in a higher level, in this case regional and the global levels, and the other looking toward parts in lower levels of SDIs such as state and local levels. This is also illustrated by a double-ended arrow in Figure 2. According to Timpf (1998), the most common function to build a hierarchy is the aggregation function. Classes of individuals are aggregated because they share a common property or attribute. This is the other reason that a hierarchical concept can be applied to SDIs. Because, different SDI initiatives at a certain political/administrative level can aggregate together to form the next higher level of hierarchy. This is the most common type of construction of hierarchy as introduced by Timpf (1998).

The existence of hierarchical capability for SDIs will enable utilisation of the advantages of this concept. Rajabifard et. al (2000) published two views on the nature of this SDI hierarchy. The first view is an umbrella view, in which the SDI at a higher level, say the global level, encompasses all the components of SDIs at levels below. This suggests that, ideally at a global level, the necessary institutional framework, technical standards, access network and people are in place to support sharing of fundamental spatial data sets kept at lower levels, such as the regional and national levels. The second view is the building block view, in which any level of SDI, say the state level, serves as 
the building block supporting the provision of spatial data needed by SDIs at higher levels in the hierarchy, such as the national or regional levels.

Based on these two views, the SDI hierarchy creates an environment, in which decision-makers working at any level can draw on data from other levels, depending on the themes, scales, currency and coverage of the data needed.

\section{HSR AND AN SDI HIERARCHY}

HSR provides an expandable framework to demonstrate the concept of SDI. Current properties of HSR theory have been particularly well adapted to describe the vertical relationships between political/administrative levels of SDIs. Additional to these vertical relationships there are complex relationships between SDIs within a political/administrative level, at an 'horizontal' level, of an SDI hierarchy. Figure 3 is a concept diagram that represents the complex vertical relationships between SDIs at levels in an SDI hierarchy $(\uparrow)$ as well as the complex horizontal relationships between SDIs in any one level of such a hierarchy $(\leftrightarrow)$. These 'horizontal' relationships have been less well explored within current HSR theory in respect to SDIs.

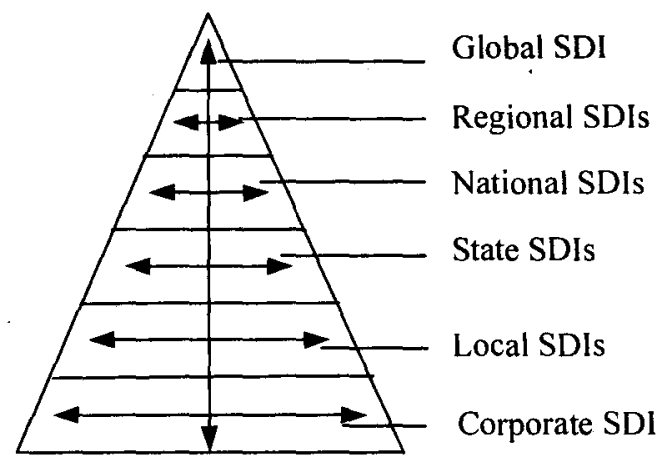

Figure 3. The complex SDI relationships within and between different levels

Recent research on SDI hierarchy has determined that current hierarchical properties, which are well utilised for modelling points and lines, are not sufficient to adequately model the complexity of the relationships between and within levels of SDIs. Therefore, to model an SDI hierarchy, the theory of HSR requires further development to incorporate the complexities of SDI relationships and dynamics.

\section{Relationships among Different SDIs}

Relationships among different levels of SDIs are complex. This complexity is due to the dynamic, inter- and intra-jurisdictional nature of SDIs. One way to observe and map these relationships in the context of an SDI hierarchy can be to assess the impact and relationships of each component of any level of SDI on the same component of an SDI at a different level. Rajabifard et al. (2000) observed the behaviour and inter-relationships between any level of SDI on the other levels through each of the components, and demonstrated a general pattern of direct and indirect potential impacts and relationships between them.

According to the pattern, a National SDI has a full impact and relationship on the other levels of the SDI hierarchy through its components. In terms of policy, National SDI have an important effect on the upper and lower levels. However, policy at a global level has only a direct impact on and relationship with Regional and National SDIs. In terms of fundamental data sets, a National SDI has an important role in forming this component of the upper levels, and its data sets are created based on 
the data sets from the lower levels of SDIs. But the fundamental data sets at a national level can have an indirect impact on the fundamental data sets at a state level. Users at a state level might need to use national fundamental data sets for their applications before using state data sets that are in more detail. In terms of technical standards, a National SDI has a direct influence on the State and Local SDIs, and its position is important for the upper levels to decide on their strategies and standards.

A national level SDI therefore, has stronger relationships as well as a more important role, in building the other levels of SDI. The role of a National SDI in an SDI hierarchy displays a particularity not present in the other levels of the SDI hierarchy. This particularity is that bottom levels of an SDI hierarchy, such as local and state, have no strong links to the upper levels of the hierarchy, like to the GSDI. So, there is a crucial level to the lower and higher links, which is the National SDI. Similar situations may exist when the first three levels (local, state and national) of an SDI hierarchy are to be considered, especially within the federated nations. In this case a State SDI is a crucial level to the local and national levels.

As mentioned above, additional to the vertical relationships between different levels of SDIs (Figure 3), there are also horizontal relationships between individual SDI initiatives within any level of an SDI hierarchy which should be taken into consideration. These relationships become more important when the respective jurisdictions are spatially adjacent and proximate. SDIs belonging to adjacent jurisdictions play more important roles and have more influence and impact on each other than on SDIs of non-adjacent jurisdictions. For example, at a regional level, the policies and standards used on preparation of fundamental data sets of country A and country B, in Figure 4, have more impact on each other than country $A$ with country $C$ or $D$, when they are supposed to be integrated together forming data sets of the region. Using a global example, the policies and standards of SDIs of the European countries have more impact on each other than they do on the policies and standards adopted for SDIs by countries from the Asia and Pacific region as an example, or Africa. This is a result of the principles of adjacency and proximity.

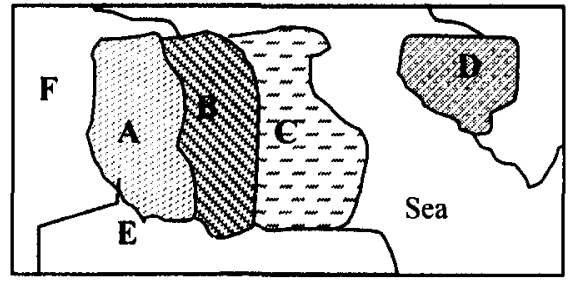

Figure 4. Countries with Adjacency and non-adjacency areas

Based on the above discussion, it is proposed that a new property must exist when applying HSR principles to SDI. This is a horizontal property which defines the levelled nature of SDI within a hierarchically organised system. This property states that within each level of the SDI hierarchy, any SDI is interconnected with another in the same level and has horizontal relationships with them in which they impact on each other.

Horizontal property encompasses the relationship between SDIs that are proximate as well as those that are distant. Coming back to the example on Figure 1, the presence of a horizontal property will make elements at a same level like I, J, K, L, Q, R, S, and T closer to each other than what they are to elements in their upper levels, even if they do not share a common intermediate upper level. In a sense, this contradicts the property of near decomposability. Further research into how HSR and its properties might be adopted into SDI is needed. 


\section{CONCLUSION}

With the increased demand for geospatial information it is proposed that the realignment of SDIs, based on HSR, will relinquish many of the present issues constraining understanding of the nature and concept of SDIs. Based on the concept, properties and reasons for using a hierarchical structure, a model of SDI hierarchy is discussed, and found suitable to apply to the concept of an SDIs' development. HSR provides an expandable framework to demonstrate the concept of SDI and represent the complexities of the different levels of SDI based on hierarchical principles.

Based on the relationships among different SDIs, one more hierarchical property, namely horizontal property, is proposed and further particularity of the SDI hierarchy is also identified. According to this property, any SDI is interconnected with the other SDIs in the same level and has horizontal relationships with them in which they impact on each other. Within interconnectivity, there is also an impact influenced by the adjacency of two areas.

The particularity within an SDI hierarchy suggests that an SDI at a national level has a crucial role in the development and implementation of the other levels of SDIs in the hierarchy. Therefore, those countries that are able to develop an efficient national SDI, will be well placed to contribute to the development of the regional and the global SDI initiatives. In this regard this paper investigated the hierarchical relationships between different political/administrative levels of SDIs and applicability of HSR as a theoretical framework to describe the multi-dimensional nature of SDIs.

\section{ACKNOWLEDGMENTS}

The authors wish to gratefully acknowledge the support of the University of Melbourne, the Australian Land Information Group (AUSLIG), the Permanent Committee on GIS Infrastructure for Asia and the Pacific (PCGIAP), and the members of the spatial data infrastructure and cadastral research group at the Department of Geomatics, the University of Melbourne, in the preparation of this paper and the associated research. However, the views expressed in the paper are those of the authors and do not necessarily reflect the views of these groups.

\section{REFERENCES}

ANZLIC. (1998) Discussion paper, Spatial Data Infrastructure for Australia and New Zealand, Accessed November 1998, <http://www.anzlic.org.au/anzdiscu.htm>

Barr, R. (1999) A private matter, GIS Europe, January: 16-17

Barr, R. (1998) I had a dream..., GIS Europe, December:16-17

Car, A. (1997) Hierarchical Spatial Reasoning: Theoretical Consideration and its Application to Modeling Wayfinding, PhD thesis, Department of Geoinformation, Technical University Vienna

Chan, T. O. and Williamson, I. P. (1999), Spatial Data Infrastructure Management: Lessons from corporate GIS development, Proceedings of AURISA'99, Blue Mountains, NSW, AURISA'99: CD-ROM

Coffey, W.J. (1981), Geography: Towards a General Spatial Systems Approach, London, New York: Methuen Co.

Coleman, D. J. and McLaughlin J., 1998, Defining global geospatial data infrastructure (GGDI): components, stakeholders and interfaces, Geomatics Journal, Canadian Institute of Geomatics, Vol. 52, No. 2, pp. 129-144

Eagleson, S., Escobar, F., and Williamson, I.P. (1999), Spatial Hierarchical Reasoning Applied to Administrative Boundary Design Using GIS, Proceedings of the Australian National Surveying Congress, Perth, Australia

Executive Order (1994), Coordinating geographic data acquisition and access, the National Spatial Data Infrastructure, Executive Order 12906, Federal Register 59, 1767117674, Executive Office of the President, USA 
European Commission, (1995), GI2000-Towards a European Geographic Information Infrastructure (EGII), A discussion document for consultation with the European GI community. European Commission, Accessed December 1999, <http://tempus1.utc.sk/gis/txts/gi2000xz.htm>

FGDC (1997), Framework, introduction and guide, Federal geographic data committee, Washington, USA

Frank, A. U. and Timpf, S. (1994), Multiple Representations for cartographic objects in a multi-scale tree - an intelligent graphical zoom, Computers and Graphics Special Issue on Modelling and Visualisation of Spatial Data in GIS

Glasgow, J. (1995), A Formalism for Model-Based Spatial Planning, In Spatial Information Theory A Theoretical Basis for GIS (International Conference COSIT'95), edited by Frank, A.U. and Kuhn, W. 988. Berlin-Heidelberg: Springer-Verlag

GSDI (1999), GSDI brochure published by AUSLIG as the Secretariat resides with Australia until the 4th GSDI Conference

Koestler, A. (1968), Das Gespesnt in der Maschine (The Ghost in the Machine), Wien-MunchenZurich: Verlag Fritz Molden

Longhorn, R. A. (2000), Regional Geographic Information Policy: Fact or Fiction - the case in Europe and lessons for GSDI, Proceedings of 4th GSDI Conference, Cape Town, South Africa

Masser, I. (1998), The first Generation of National Geographic Information Strategies, Proceedings of Selected Conference Papers of the Third Global Spatial Data Infrastructure Conference, November 1998, Canberra

McLaughlin, J.D., and Nichols, S.E. (1992), Building a national spatial data infrastructure, Proceedings of Computing Canada, 6th January: 24

Mesarovic, M.D., Macko, D., and Takahara, Y. (1970), Theory of hierarchical, multilevel, systems, Mathematics in science and engineering, Ed. Bellmann, Richard. New York: Academic Press

Mohamed, A. M. (1999), PCGIAP and the Asia-Pacific Spatial Data Infrastructure (APSDI), Proceedings of the Cambridge Conference, Cambridge, 1999

Onsrud, H. (1998), Survey of national and regional spatial data infrastructure activities around the globe, Proceedings of Selected Conference Papers of the Third Global Spatial Data Infrastructure Conference, November 1998, Canberra

Pattee, H.H. (1973), Hierarchy Theory - The Challenge of Complex Systems, New York: Braziller Rajabifard, A., Williamson, I.P., Holland, P., and Johnstone, G. (2000), From Local to Global SDI initiatives: a pyramid building blocks, Proceedings of the 4th GSDI Conference, Cape Town, South Africa, <http://www.gsdi.gov. docs.html>

Simon, H.A. (1981), The Sciences of the Artificial, Cambridge, MA: MIT Press

Simon, H.A. (1973), The Organisation of Complex Systems, In Hierarchy Theory, Pattee H. (Eds.), pp. 1-27 (New York: Braziller)

Timpf, S. (1998), Hierarchical Structures in Map Series, PhD thesis, Department of Geoinformation, Technical University Vienna

Timpf, S. and Frank, A.U. (1997), Using hierarchical spatial data structures for hierarchical spatial reasoning, Spatial Information Theory - A Theoretical Basis for GIS (International Conference COSIT'97), edited by Hirtle, Stephen C. and Frank, Andrew U. Lecture Notes in Computer Science 1329. Berlin-Heidelberg: Springer-Verlag

Timpf, S., Volta, G.S., Pollock, D.W., and Egenhofer, M.J. (1992), A Conceptual Model of Wayfinding Using Multiple Levels of Abstractions, In Theories and Methods of SpatioTemporal Reasoning in Geographic Space, edited by Frank, A.U., Campari, I., and Formentini, U. 639. Heidelberg-Berlin: Springer Verlag

Volta, G., and Egenhofer, M. (1993), Interaction with GIS Attribute Data Based on Categorical Coverages, in: A. Frank and I. Campari (eds.), European Conference on Spatial Information Theory, Marciana Marina, Italy 